\title{
The Electroluminescent Decay Mechanism of Rare-Earth Ions in OLEDs Based on a Terbium Complex
}

\author{
C. J. Liang, Wallace C. H. Choy, and ChunHui Huang
}

\begin{abstract}
The organic light-emitting diodes (OLEDs) based on terbium ( $\mathbf{T b})$ complexes show sharp green emission spectrum with excellent color purity. However, the brightness of Tb-OLEDs is generally weak. Here, the electroluminescent (EL) decay mechanism of TB-OLEDs is studied by arbitrarily using tris-(1-phenyl-3methyl-4-isobutyryl-5-pyrozolone)-bis(triphenyl phosphine oxide) terbium as the emitting layer. The device shows high EL efficiency at low current density but rapid reduction of device efficiency at higher current density. The transient EL is investigated for understanding the decay process of excited $\mathrm{Tb}^{3+}$ ions. Together with theoretical studies, exciton quenching is proposed to explain the decay of the Tb-OLEDs which is important for optimizing and engineering the material and device structures. The EL from the mixed layer of the $\mathrm{Tb}$ and europium (Eu) complexes is also studied. We find that the EL performance and transient decay of the excited $\mathrm{Tb}$ ions are modified by energy transfer from $\mathrm{Tb}$ to $\mathrm{Eu}$ in the OLEDs.
\end{abstract}

Index Terms-Electroluminescent (EL) decay mechanism, energy transfer, exciton quenching, rare-earth (RE) organic lightemitting diodes (OLEDs).

\section{INTRODUCTION}

$\mathbf{I}$ T IS well-known that rare-earth (RE) ions can exhibit spectrally narrow emission, due to intra-atomic transitions within the $4 f$ shell. Earlier photoluminescence (PL) studies showed that RE complexes have the interesting features of highly efficient intra-energy conversion from the singlet states of organic ligands to the triplet states and thence to the excited states of the central RE ions; thus, both singlet and triplet excited states of the ligands could contribute to the final sharp emission of the central RE ions for device applications. Therefore, with a proper engineering of the material and device structures, RE complexes are anticipated to exhibit high efficiencies in organic electroluminescent (EL) devices. For these reasons, there has been great interest in using RE complexes as light emitters in the organic light-emitting diodes (OLEDs). So far, the EL from the RE complexes of Eu, Tb, Dy, and Sm [1]-[4] for visible emission, and $\mathrm{Er}, \mathrm{Nd}$, and $\mathrm{Yb}[5]-[7]$ for infrared emission have been realized. However, the experimental results show that the EL efficiency

Manuscript received December 28, 2006; revised April 11, 2007. This work was supported by a UDF grant, by the Strategic Research Grant in Organic Optoelectronics of Hong Kong University, and by Grant HKU 7124/05E from the Research Grant Council of the Hong Kong Special Administrative Region, China.

C. J. Liang and W. C. H. Choy are with the Department of Electrical and Electronic Engineering, University of Hong Kong, Hong Kong (e-mail: chchoy@eee.hku.hk).

C. Huang is with the State Key Laboratory of Rare Earth Materials Chemistry and Applications, Peking University, Beijing 100871, China.

Digital Object Identifier 10.1109/LPT.2007.901599 and the emission intensity of the RE complex-based OLEDs are much lower than the expected. This remains to be the inherent feature for the application of RE complexes in OLEDs. For a better understanding of the issue, it is necessary to investigate the excited-states process of the RE ions in OLEDs [7].

$\mathrm{Eu}$ and $\mathrm{Tb}$ complexes are the most intensively investigated RE complexes in OLEDs. Eu complexes have shown high EL efficiency at low current density but rapid reduction of device efficiency at higher current density. It has been reported [1], [8], [9] that in the Eu-based EL device, the exciton quenching among the excited $\mathrm{Eu}^{3+}$ ions is an important channel in the decay process, and this is the primary cause for the rapid reduction of emission efficiency at higher excitation intensity. For Tb-based OLED, the EL performances of $\mathrm{Tb}$ complexes with various organic ligands have shown that the overall EL intensity and efficiency are not good, and improvements have been investigated [10]-[13]. However, the decay mechanism of the Tb-based OLED remains to be a question. It is necessary to clarify the excited-states process of the $\mathrm{Tb}$ complex in the OLEDs.

In this letter, we examine the EL performance and decay mechanism of the OLEDs using tris-(1-phenyl-3-methyl-4isobutyryl-5-pyrozolone)-bis(triphenyl phosphine oxide) $\left(\mathrm{Tb}(\mathrm{PMIP})_{3}(\mathrm{TPPO})_{2}\right)$ as the emitting layer. The results suggest that exciton quenching occurs in the Tb-based OLEDs. We also investigated the EL from the mixed layer of the Tb complex and a Eu complexes of europium (dibenzoylmethanato) 3 (bathophenanthroline) $\left(\mathrm{Eu}(\mathrm{DBM})_{3}\right.$ bath). The results show that energy transfer from $\mathrm{Tb}$ to Eu ions occurs in the mixed layer and modify the emission decay of $\mathrm{Tb}$.

\section{EXPERIMENT}

$\mathrm{Tb}(\mathrm{PMIP})_{3}(\mathrm{TPPO})_{2}$ and a mixed layer of the $\mathrm{Tb}$ complex and $\mathrm{Eu}(\mathrm{DBM})_{3}$ bath were used as the emitting layers in this study. The widely used material of $N, N^{\prime}$-Bis (3-methylphenyl)- $N, N^{\prime}$-diphenyl-benzidine (TPD) is used as the hole-transport layer. UV emissive $2,2^{\prime}, 2^{\prime \prime}-(1,3,5$-Benzinetriyl)-tris (1-phenyl-1-H-benzimidazole) (TPBI) is the electron-transport layer. The OLEDs with the structure of

Device A: ITO-TPD(50 nm)/Tb-complex(30 $\mathrm{nm}) /$ $\mathrm{TPBI}(30 \mathrm{~nm}) / \mathrm{LiF}-\mathrm{Al}$, and

Device B: ITO-TPD(50 nm)/Tb-complex: Eucomplex(30 nm)/TPBI(30 nm)/LiF-Al

were fabricated. All the organics and the $\mathrm{LiF}-\mathrm{Al}$ cathode are deposited by thermal vacuum deposition at a pressure of $10^{-6}$ Torr [14]. Transient EL was studied by applying a voltage pulse across the device. By using a monochromator for 


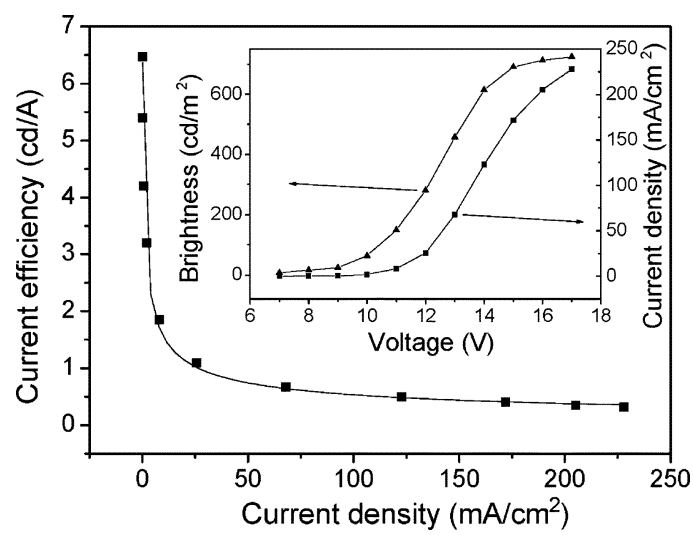

Fig. 1. Efficiency-current characteristics of Device A: experimental data (filled squares) and simulated data (solid line) by using (2), with $\eta_{0}=7.4 \mathrm{~cd} / \mathrm{A}$ and $j_{0}=1.0 \mathrm{~mA} / \mathrm{cm}^{2}$. The inset shows brightness-current-voltage characteristics of Device A.

wavelength selection, the transient spectrum was detected by a photomultiplier and an oscilloscope.

\section{RESULTS AND DISCUSSION}

Fig. 1 shows the efficiency-current relationship of Device A. It shows high EL efficiency of $6.5 \mathrm{~cd} / \mathrm{A}$ at the low current density of $0.1 \mathrm{~mA} / \mathrm{cm}^{2}$. However, the EL efficiency drops rapidly with the current density. It reduces to below $1.0 \mathrm{~cd} / \mathrm{A}$ when the current density is higher than $30 \mathrm{~mA} / \mathrm{cm}^{2}$. As a result, the device cannot exhibit high EL brightness as shown in the inset of Fig. 1. By simultaneously investigating the experimental results with the theoretical model, we clarify the emission decay mechanism. In the exciton quenching model, it is assumed that the interaction between two excited $\mathrm{Tb}^{3+}$ ions causes a quenching channel in the decay process of the excited $\mathrm{Tb}^{3+}$ ions, thus the process will follow:

$$
\frac{d n}{d t}=-\frac{n}{\tau}-\frac{k_{q}}{2} n^{2}+k_{f} \frac{j}{q l}
$$

where $n$ is the concentration of the excited $\mathrm{Tb}^{3+}$ ions, $\tau$ is the lifetime of the excited ions due to emissive decay, $k_{q}$ is the exciton quenching parameter, $j$ is the current density, $q$ is the electron charge, $l$ is the thickness of the exciton formation zone, and $k_{f}$ is the possibility for a charge-carrier to form an excited $\mathrm{Tb}^{3+}$ ion. At steady state, the quantum efficiency can be calculated from (1)

$$
\eta=\eta_{0} \frac{j_{0}}{4 j}\left(\sqrt{1+8 \frac{j}{j_{0}}}-1\right)
$$

where $\eta$ is the device efficiency, $\eta_{0}$ is the efficiency in the absence of the biexcitonic quenching effect among the excited $\mathrm{Tb}^{3+}$ ions, and

$$
j_{0}=4 l q /\left(k_{q} k_{f} \tau^{2}\right)
$$

is the characteristic current density at which $\eta=\eta_{0} / 2$. If $j_{0}$ were small, the quenching effect would be serious. Thus, a large value of $j_{0}$ is expected in the devices. The transient decay process could be deduced from (1). After a pulse excitation, the emission intensity $L(t)$ follows:

$$
L(t)=L(0) /\left[(1+K \tau) e^{\frac{t}{\tau}}-K \tau\right]
$$

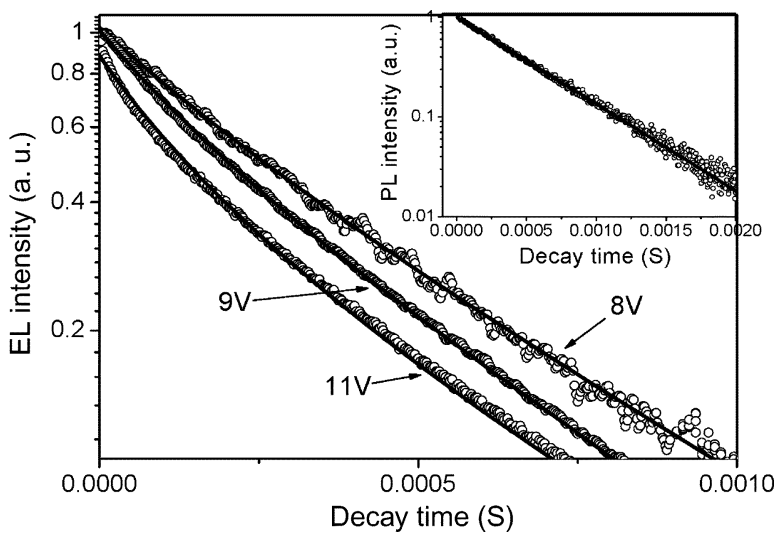

Fig. 2. Transient luminescence of Device A after the pulse excitations at 8, 9, and $11 \mathrm{~V}$ : experimental data (open circles) and fitting curves (solid line) by using equation (4) with $1.1 \times 10^{3}, 2.0 \times 10^{3}$, and $3.1 \times 10^{3} \mathrm{~s}^{-1}$ for $K$ at 8 , 9, and $11 \mathrm{~V}$, respectively. The fitting curves are normalized at $t=0$ for comparison. The inset shows the decay time of $\mathrm{Tb}(\mathrm{PMIP})_{3}(\mathrm{TPPO})_{2}$ characterized by PL measurements.

where $L(0)$ is the initial emission intensity and $K$ is defined by

$$
K=0.5\left[k_{q} n(0)\right] .
$$

Here $n(0)$ is the initial concentration of the excited $\mathrm{Tb}^{3+}$ ions.

We are ready to make comparison between the theory and the experimental results. As shown in Fig. 1, the experimental data on the efficiency-current relationship are well fitted with the simulation line based on (2). In the computer-assisted simulation, the values $\eta_{0}=7.4 \mathrm{~cd} / \mathrm{A}$ and $j_{0}=1.0 \mathrm{~mA} / \mathrm{cm}^{2}$ were used. This means that if the current density is higher than $1.0 \mathrm{~mA} / \mathrm{cm}^{2}$, the efficiency will be less than half of the initial value. The value of $k_{q}$ is estimated to be $2.7 \times 10^{-21} \mathrm{~m}^{3} / \mathrm{s}$ according to (3) by using $j_{0}=1.0 \mathrm{~mA} / \mathrm{cm}^{2}, l=10 \mathrm{~nm}, \tau=0.49 \mathrm{~ms}$, and $k_{f}=1$.

More straightforward evidence supporting the exciton quenching model is the transient EL signal of the device. Fig. 2 shows the decay process after excitation with a pulse voltage. The measurement was performed at pulse voltages of 8,9 , and $11 \mathrm{~V}$. The decay becomes faster with increasing voltages, and the measured transient luminescence could not simply be described by a single exponential decay rule, especially at higher voltage, suggesting a nonlinear quenching term in the decay process. The inset of Fig. 2 shows PL measurements on the decay time of the $\mathrm{Tb}$ complex. The exponential decay fit on this data gives a lifetime of $0.49 \mathrm{~ms}$. Using this value for $\tau$ in (4), the decay process is fitted as shown in Fig. 2. The values of $K$ are $1.1 \times 10^{3}, 2.0 \times 10^{3}$, and $3.1 \times 10^{3} \mathrm{~s}^{-1}$ for 8,9 , and $11 \mathrm{~V}$, respectively. The $K$ values increase with increasing voltages. According to (5), this trend is reasonable because a higher voltage pulse would give a higher initial concentration $n(0)$ and thus a larger $K$. Consequently, the theoretical and experimental results suggest that exciton quenching occurs in the $\mathrm{Tb}$ complex-based OLEDs.

In order to study the interaction between $\mathrm{Tb}$ and Eu ions, Device B with a mixed layer of the Tb complex and Eu complex has been studied. Fig. 3 shows the EL spectra of the device with different weight ratio of the $\mathrm{Eu}$ and $\mathrm{Tb}$ complexes. For reference, EL from the pure Tb complex is shown in Fig. 3(A). In Fig. 3(B), the weight ratio of Eu and Tb is $1: 15$; the Eu emission at $613 \mathrm{~nm}$ appears in the spectrum. When the $\mathrm{Eu}: \mathrm{Tb}$ ratio is increased to $3: 15$, the emission from Eu dominates the EL 


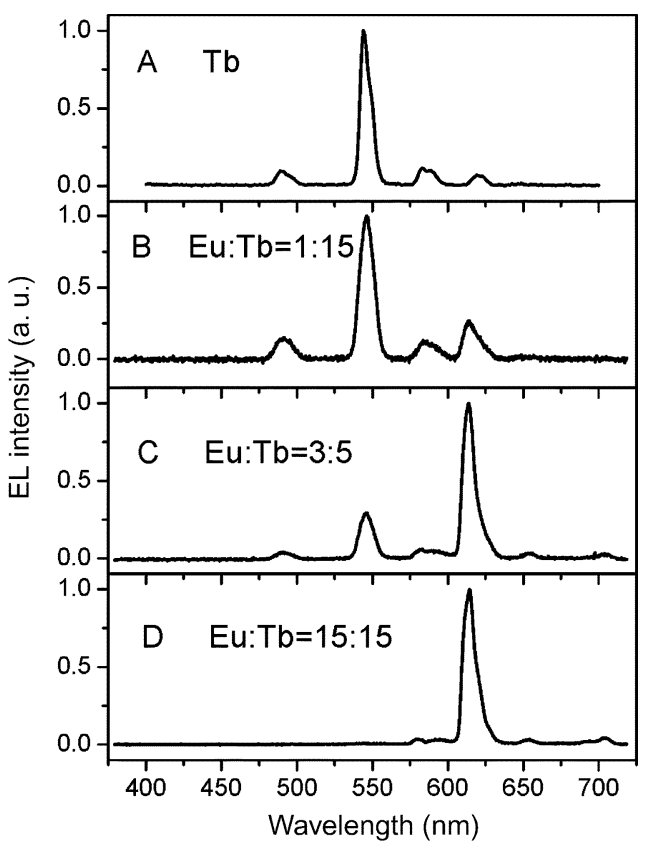

Fig. 3. EL spectra of the device with different ratio of the Eu and Tb complex, the Eu-to-Tb weight ratio of the mixed layer are $0: 15$ (pure Tb complex), $1: 15$, and $3: 15,15: 15$, respectively.

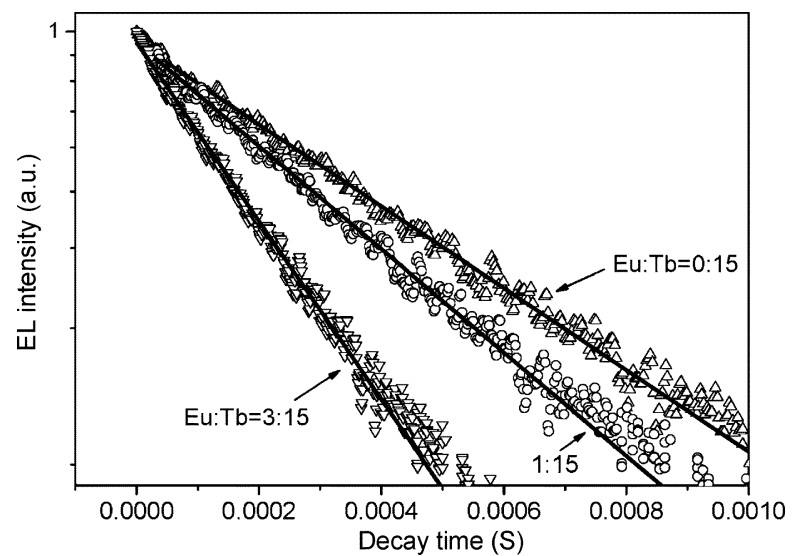

Fig. 4. Transient decay curve of $\mathrm{Tb}^{3+}$ ions in the mixing layer after excitation with pulse voltages of $6.8 \mathrm{~V}$, the Eu-to-Tb weight ratio of the mixed layer are $0: 15$ (pure Tb complex), $1: 15$, and $3: 15$, respectively.

spectra as shown in (C). In Fig. 3(D), the Eu: Tb ratio is further increased to $15: 15$; in this case, only emission from Eu can be seen in the EL spectrum, and the Tb emission is totally disappeared. These results show that the existence of the Eu ions in the mixed layer suppress the emission of $\mathrm{Tb}$ ions, suggesting that energy transfer from $\mathrm{Tb}$ to Eu may happen in the mixed layer.

Transient EL measurement of the $\mathrm{Tb}$ emission is performed to confirm the prediction. The decay of $\mathrm{Tb}^{3+}$ ions in the mixing layer after excitation with pulse voltages are shown in Fig. 4. We set the measured wavelength at $545 \mathrm{~nm}$ such that the detected signals only describe the decay of $\mathrm{Tb}^{3+}$ ions. The excitation intensity is monitored as low as possible, so that the exciton quenching are negligible and the decay signal can be fitted with an exponential decay rule. The transient measurements are made at the $\mathrm{Eu}: \mathrm{Tb}$ ratio of $0: 15$ (pure Tb complex), $1: 15$, and $3: 15$. The exponential decay fit on the data gives a decay lifetime of $0.49,0.39$, and $0.22 \mathrm{~ms}$ for the ratio of $0: 15,1: 15$ and $3: 15$, respectively. This shows that the existence of Eu changes the decay rate of $\mathrm{Tb}^{3+}$ significantly; the more the Eu complex in the mixing layer, the faster the decay of $\mathrm{Tb}^{3+}$ will be. This indicates the energy transfer from $\mathrm{Tb}$ to Eu ions introduces another decay channel for excited $\mathrm{Tb}^{3+}$ ions and accelerates its decay process.

\section{CONCLUSION}

Because the excited RE ions possess very long lifetime, the interaction between the ions at their excited states becomes significant. Similar to the EL of Eu complex, the exciton quenching also occurs in the Tb complex-based OLEDs. This quenching process is a primary cause for the efficiency reduction and low emitting intensity of the device. Interaction between different RE ions is also observed. The results on EL spectrum performance and transient decay of $\mathrm{Tb}$ ions in the mixed layer of $\mathrm{Tb}$ complex and Eu complex show that the energy transfer from $\mathrm{Tb}$ to Eu introduces another decay channel for excited $\mathrm{Tb}^{3+}$ ions and accelerates its decay process.

\section{REFERENCES}

[1] C. J. Liang and W. C. H. Choy, "Color-tunable organic light-emitting diodes by using europium organometallic complex," Appl. Phys. Lett., vol. 89, pp. 251108-1-251108-3, 2006.

[2] X. C. Gao, H. Cao, C. H. Huang, B. G. Li, and S. Umitani, "Electroluminescence of a novel terbium complex," Appl. Phys. Lett., vol. 72, no. 18, pp. 2217-2219, 1998.

[3] Z. R. Hong, W. L. Li, D. X. Zhao, C. J. Liang, X. Y. Liu, J. B. Peng, and D. Zhao, "White light emission from OEL devices based on organic dysprosium complex," Synth. Met., vol. 111-112, pp. 43-45, 2000.

[4] E. Stathatos, P. Lianos, E. Evgeniou, and A. D. Keramidas, "Electroluminescence by a $\mathrm{Sm}^{3+}$-diketonate-phenanthroline complex," Synth. Met., vol. 139, pp. 433-437, 2003.

[5] R. J. Curry and W. P. Gillin, "Infra-red and visible electroluminescence from ErQ based OLEDs," Synth. Met., vol. 111-112, pp. 35-38, Jun. 2000.

[6] O. M. Khreis, R. J. Curry, M. Somerton, and W. P. Gillin, "Infrared organic photolight emitting diodes using neodymium tris-(8-hydroxyquinoline)," J. Appl. Phys., vol. 88, no. 2, pp. 777-780, 2000.

[7] O. M. Khreis, W. P. Gillin, M. Somerton, and R. J. Curry, " $980 \mathrm{~nm}$ electroluminescence from ytterbium tris (8-hydroxyquinoline)," Organic Electron., vol. 2, no. 1, pp. 45-51, 2001.

[8] C. J. Liang, T. C. Wong, L. S. Hung, S. T. Lee, Z. R. Hong, and W. L. $\mathrm{Li}$, "Self-quenching of excited europium ions in Eu(DBM)3bath-based organic electroluminescent devices," J. Phys. D: Appl. Phys., vol. 34, pp. L61-L64, 2001.

[9] T. W. Canzler and J. Kido, "Exciton quenching in highly efficient europium-complex based organic light-emitting diodes," Organic Electron., vol. 7, pp. 29-37, Feb. 2006.

[10] D. Tao, Y. Xu, J. Feng, T. Zhang, Z. Xu, H. Shen, J. Wu, Z. Xu, X. $\mathrm{Xu}$, and $\mathrm{D}$. $\mathrm{Xu}$, "Using a terbium complex as an energy bridge to enhance energy transfer between polyvinyl carbazole and europium complexes," J. Mater. Chem., vol. 14, pp. 1252-1256, 2004.

[11] Y. X. Zheng, J. Lin, Y. J. Liang, Q. Lin, Y. N. Yu, C. Guo, S. B. Wang, and H. J. Zhang, "A novel terbium (III) beta-diketonate complex as thin film for optical device application," Mater. Lett., vol. 54, no. 5-6, pp. 424-429, 2002.

[12] X. C. Gao, H. Cao, C. H. Huang, S. Umitani, G. Q. Chen, and P. Jiang, "Photoluminescence and electroluminescence of a series of terbium complexes," Synth. Met., vol. 99, pp. 127-132, Feb. 1999.

[13] S. Capecchi, O. Renault, D. G. Moon, M. Halim, M. Etchells, P. J. Dobson, O. V. Salata, and V. Christou, "High-efficiency organic electroluminescent devices using an organoterbium emitter," Adv. Mater., vol. 12, pp. 1591-1598, Nov. 2000.

[14] H. H. Fong, W. C. H. Choy, K. N. Hui, and Y. J. Liang, "Organic lightemitting diodes based on a co-host electron transporting composite," Appl. Phys. Lett., vol. 88, pp. 113510-1-113510-3, 2006. 